\title{
Quality control of lymph node dissection in the Dutch randomized trial of D1 and D2 lymph node dissection for gastric cancer
}

\author{
J.J Bonenkamp ${ }^{3}$, J. Hermans ${ }^{2}$, M. Sasako ${ }^{4}$, and C.J.H. van de Velde ${ }^{1}$ \\ Departments of ${ }^{1}$ Surgery and ${ }^{2}$ Medical Statistics, Leiden University Medical Center, PO Box 9700, 2300 RC Leiden, The Netherlands \\ ${ }^{3}$ Hospital of the Free University of Amsterdam, The Netherlands \\ ${ }^{4}$ National Cancer Center Hospital of Tokyo, Tokyo, Japan
}

\begin{abstract}
:
Background. Variability among surgeons and reduced protocol adherence threaten the conduct and outcome of surgical multicenter trials. We introduced, in the Dutch Gastric Cancer Trial of D1 and D2 (extended) lymph node dissection for gastric cancer, a novel way of managing instruction, quality control, and evaluation of protocol adherence.

Methods. Of 1078 patients entered in the Dutch trial, 711 patients with potentially curative resections were evaluated. Numbers and locations of lymph nodes detected at pathological investigation were compared according to the guidelines of the Japanese Research Society for the Study of Gastric Carcer. Non-compliance indicated inadequate removal of lymph node stations, whereas contamination indicated that lymph nodes were detected outside the intended level of dissection. Protocol adherence during the course of the trial, and the impact on complications, hospital mortality, and survival were evaluated.

Results. Major non-compliance was noted in $15.3 \%$ of D1 and $25.9 \%$ of $\mathrm{D} 2$ patients. Contamination was present in $22.9 \%$ of D1 and $23.5 \%$ of D2 patients, and was limited to one or two lymph node stations only. Intensification of quality control resulted in only a marginal improvement in protocol adherence and in the number of lymph nodes detected. There was no association between protocol adherence and the occurrence of complications or long term survival.

Conclusions. Contamination proved an important parameter to substantiate protocol adherence by the surgeon, whereas non-compliance had a multifactorial cause. Non-adherence to the protocol did not lead to increased hospital morbidity and mortality, but also had no impact on long term survival.
\end{abstract}

Key words: gastric cancer, lymph node dissection, randomized trial, quality control

Offprint requests to: C.J.H. van de Velde Received for publication on Aug. 17, 1998; accepted on Nov. 12, 1998

\section{Introduction}

Despite the increasing worldwide interest in extended (D2) lymph node dissection for gastric cancer there is still reluctance to test its value in prospective randomized studies. The high-incidence countries, notably Japan, have adopted extended lymph node dissection as the routine operation for gastric cancer and it is now so widely accepted that, in their view, a randomized trial to demonstrate the disadvantage of limited (D1) dissection would be unethical. Hospital morbidity and mortality are extremely low in Japan, and even dissections wider than D2 can be done with limited morbidity [1].

In Western countries, on the other hand, the fear of complications has prevented the general use of D2 dissection. In the only two published large randomized trials of extended lymph node dissection, postoperative complication rates proved unacceptably high $[2,3]$. The technique has been introduced in centers specialized in oncological surgery, and the increased morbidity has not been seen there [4-6]. In order to detect a significant survival difference in a randomized trial, however, more patients are required than the numbers seen in one institution. Multi-center cooperation allows sufficient accrual, but has the disadvantage of introducing inter-surgeon variability in the conduct of extended lymph node dissection. This is not an imaginary danger, considering the rather complex criteria of the Japanese Research Society for the study of Gastric Cancer (JRSGC) [7]. Intra-surgeon variability should be avoided as well; surgeons less familiar with the experimental technique are more likely to show incomplete dissection and to have higher complication rates than with the conventional technique. In order to counteract these sources of variability, quality control of surgery is essential [8]. In this article, we study the effect of quality control in the Dutch Gastric Cancer Trial [3]. 


\section{Patients and methods}

From September 1989 until June 1993, 1078 patients with gastric cancer were entered in a multicenter randomized trial of D1 and D2 lymph node dissection in The Netherlands. Prior to application of the complex Japanese criteria in daily practice, participating Dutch surgeons and pathologists were instructed by a Japanese surgeon experienced in the technique of lymph node dissection [9]. Furthermore, an instruction videotape and a booklet with step-by-step photo instructions were distributed to the participants (Table 1 ). To maintain the standard achieved by this intensive instruction, peroperative guidance by regional referent surgeons for D2 dissections, and by the study coordinator for D1 dissections was instituted.

To enable these referents to attend their assigned operations, randomization had to precede the operation. At laparotomy, staging was monitored and curability determined. If signs of non-curability were present (metastases to peritoneum, liver or distant lymph nodes, or locally irresectable tumor), the patient was classified as non-curative and underwent a palliative operation regarded as appropriate by the surgeon. Patients without evidence of metastases were regarded as resectable with curative intent and underwent the allocated resection, D1 or D2. During the operation, the referent surgeons guided the extent of lymph node dissection. After resection of the stomach and the adjacent lymph nodes, they either divided the fatty tissue and verified that the lymph node-containing tissues were placed in correctly numbered containers, which were sent to the pathologist for lymph node retrieval, or they retrieved the lymph nodes one-by-one themselves in the operating theater. Only the specimens of the curatively resected patients were used for the analysis of protocol adherence. Pathologists from 55 participating laboratories processed the resection specimens and reported the number of lymph nodes retrieved per station, as well as

Table 1. Measures of quality control in the Dutch Gastric Cancer Trial (DGCT)

\begin{tabular}{|c|c|}
\hline Phase & Quality control measures \\
\hline \multirow[t]{3}{*}{ Instruction } & Instruction booklet \\
\hline & $\begin{array}{l}\text { Videotape of D2 (extended) lymph node } \\
\text { dissection }\end{array}$ \\
\hline & $\begin{array}{l}\text { Japanese surgeon in the operating theatre } \\
\text { (approximately } 50 \text { cases) }\end{array}$ \\
\hline \multirow[t]{4}{*}{ Intake } & Referent surgeon in the operating theater \\
\hline & Coordinator in the operating theater \\
\hline & Assessment of quantitative parameters \\
\hline & $\begin{array}{l}\text { Adjustment of measures to improve } \\
\text { discrimination between D1 and D2 }\end{array}$ \\
\hline Evaluation & $\begin{array}{l}\text { Verification of accuracy of the quantitative } \\
\text { parameters }\end{array}$ \\
\hline
\end{tabular}

the number of tumor-containing lymph nodes, using standard forms distributed by the trial office. All patients were staged according to the 1987 version of the International Union Against Cancer (UICC)-TNM classification system for gastric cancer [10], except for definition of the lymph node levels and the individual lymph node stations, for which the Japanese criteria were used [7].

In addition to peroperative guidance, we developed quantitative parameters to measure protocol adherence. These were based on the numbers and location of lymph nodes reported by the pathologist. Using the schedule of the JRSGC, the indication for lymph node groups can be assessed. Protocol adherence was thus evaluated on the basis of non-compliance (i.e., no yield of lymph nodes from indicated stations) and contamination (i.e., finding one or more lymph nodes in stations for which dissection was not indicated) (Fig. 1). In order to adjust the model for practical use, two assumptions were needed. Firstly, all lymph node stations were assumed to contain at least one lymph node, which was realistic in relation to published reference values for the number of nodes per station [11]. Secondly, within the Japanese criteria, dissection of "optional" stations depends on the type of stomach resection, but for other stations, there is no correction factor. For a tumor in the middle third of the stomach it is optional to do a total gastrectomy, and subsequently dissect stations number 2 (left para-cardial) and 10 (splenic hilus). On the other hand, for cancers in the proximal third, the criteria provide no alternative for proximal gastrectomy, a procedure that is not used in The Netherlands. We therefore adjusted the model for the type of stomach resection in these cases.

The quality control system was used throughout the intake phase of the trial (September 1989 to July 1993). In order to substantiate the effect of this quality control, an interim analysis of contamination and noncompliance was done on January 1, 1991. The results of this analysis forced us to intensify the quality control, in order to maintain the required distinction between the two treatment groups. The special measures, taken to minimize protocol deviations in the second-intake period were: intensified peroperative monitoring of

\begin{tabular}{|c|c|c|c|}
\hline \multicolumn{2}{|c|}{} & \multicolumn{2}{c|}{ Indication for dissection of station } \\
\cline { 2 - 4 } Protocol Deviations & Yes & No \\
\hline \multirow{2}{*}{$\begin{array}{c}\text { Lymph node yield } \\
\text { per station }\end{array}$} & 0 & Non-compliance & No Contamination \\
\cline { 2 - 4 } & $\geq 1$ & Compliance & Contamination \\
\hline
\end{tabular}

Fig. 1. Protocol deviations in the Dutch trial 
lymphadenectomy by the surgical study coordinator and the reference surgeons; lymph node retrieval immediately after the operation, both in D1 and D2, by one of the authors (J.J.B); scoring of the number of yielded nodes to disentangle surgical and pathological sources of non-compliance; evaluation of protocol deviations, and discussing of the results in each surgery-pathology team. The effect of this intensified monitoring was assessed by comparing contamination and noncompliance in two periods; period I (patients entered before January 1,1991) and period II (patients entered after January 1, 1991).

Nodal yields per Lymph node level were calculated to estimate a so-called learning effect of lymph node dissection, assuming that increasing experience would improve nodal clearance. In order to determine the validity of the assumption that all stations were expected to contain at least one lymph node, nodal yields per station were calculated as well.

To evaluate whether protocol deviations influenced the postoperative sequelae, we investigated the incidence of complications and the long-term survival in patients with and without protocol deviations.

Table 2. Number of patients entered in the Dutch Gastric Cancer Trial

\begin{tabular}{lrrr}
\hline & \multicolumn{2}{c}{ Dissection } & \\
\cline { 2 - 3 } & D1 & D2 & Total \\
\hline Randomized & 539 & 539 & 1078 \\
Ineligible & 26 & 56 & 82 \\
Eligible & 513 & 483 & 996 \\
Non-curative & 133 & 152 & 285 \\
Curative & 380 & 331 & 711 \\
\hline
\end{tabular}

To evaluate the hypothesis concerning equality of the magnitude (number of stations per patient) of protocol deviations between D1 and D2, and the incidence of non-compliance and contamination among the reference surgeons supervising the D2 resections, the $\chi^{2}$ test was used, with a significance level of 0.05 . Statistical analysis of survival differences was done by the life table method.

\section{Results}

Between September 1989 and July 1993, 1078 patients were entered at the trial data center (Table 2). Eightytwo patients did not comply with the inclusion criteria. In 30 cases, ineligibility was caused by the inability of the referent surgeon to attend the D2 operation. Eighteen patients required adjustment of the preoperative diagnosis, due to lymphoma $(n=10)$, dysplasia $(n=4)$, and pancreas carcinoma $(n=2)$; in two patients, no proof of adenocarcinoma could be found in the specimen, although the biopsy diagnosis was correct at second evaluation. At laparotomy, $133(26 \%)$ patients in the D1 group and $152(31 \%)$ in the D2 group were judged non-curative and underwent palliative treatment (53\%, palliative resection; $19 \%$, bypass; and $27 \%$, exploration only).

All other patients had the allocated type of resection, D1 $(n=380)$ or D2 $(n=331)$. Information on the number and distribution of lymph nodes was obtained from the trial forms; there were no missing cases, but in three patients, the number of lymph nodes was not available. Overall, non-compliance occurred in $80.6 \%$ of the D1 and $81.5 \%$ of the D2 patients (Table 3). The magnitude of non-compliance differed between D1 and

Table 3. Magnitude of (non)-compliance per patient; curative patients $(n=711)$

\begin{tabular}{|c|c|c|c|c|}
\hline \multirow{3}{*}{$\begin{array}{l}\text { (number of lymph node stations without a } \\
\text { yield, althougy indicated for dissection) }\end{array}$} & \multicolumn{4}{|c|}{ Dissection } \\
\hline & \multicolumn{2}{|c|}{ D1 $(n=380)$} & \multicolumn{2}{|c|}{$\mathrm{D} 2(n=331)$} \\
\hline & $n$ & $\%$ & $n$ & $\%$ \\
\hline Compliance & 74 & 19.5 & 61 & 18.4 \\
\hline Minor non-compliance & 248 & 65.3 & 184 & 55.6 \\
\hline 1 & \multicolumn{2}{|c|}{169} & \multicolumn{2}{|c|}{101} \\
\hline 2 & \multicolumn{2}{|c|}{79} & \multicolumn{2}{|c|}{83} \\
\hline Major non-compliance & 58 & 15.3 & 86 & 25.9 \\
\hline 3 & \multicolumn{2}{|c|}{37} & \multicolumn{2}{|c|}{44} \\
\hline 4 & \multirow{2}{*}{\multicolumn{2}{|c|}{$\begin{array}{r}12 \\
9\end{array}$}} & \multicolumn{2}{|c|}{19} \\
\hline 5 & & & \multicolumn{2}{|c|}{15} \\
\hline 6 & & & \multicolumn{2}{|c|}{4} \\
\hline 7 & & & \multicolumn{2}{|c|}{1} \\
\hline 9 & & & \multicolumn{2}{|c|}{2} \\
\hline 11 & & & \multicolumn{2}{|c|}{1} \\
\hline Overall non-compliance & 306 & 80.6 & 270 & 81.5 \\
\hline
\end{tabular}


Table 4. Magnitude of contamination per patient; curative patients $(n=711)$

\begin{tabular}{|c|c|c|c|c|}
\hline \multirow{3}{*}{$\begin{array}{l}\text { (number of lymph node stations reported, } \\
\text { although indicated for dissection) }\end{array}$} & \multicolumn{4}{|c|}{ Dissection } \\
\hline & \multicolumn{2}{|c|}{$\mathrm{D} 1(n=380)$} & \multicolumn{2}{|c|}{$\mathrm{D} 2(n=331)$} \\
\hline & $n$ & $\%$ & $n$ & $\%$ \\
\hline No contamination & 282 & 74.2 & 236 & 71.3 \\
\hline Minor contamination & 95 & 25.0 & 95 & 28.7 \\
\hline 1 & \multicolumn{2}{|c|}{76} & \multicolumn{2}{|c|}{72} \\
\hline 2 & \multicolumn{2}{|c|}{19} & \multicolumn{2}{|c|}{23} \\
\hline Major contamination & 3 & 0.8 & 0 & 0.0 \\
\hline 3 & 1 & & & \\
\hline 4 & 2 & & & \\
\hline Overall contamination & 98 & 25.8 & 95 & 28.7 \\
\hline
\end{tabular}

Table 5. Magnitude of (non)-compliance and contamination for curative patients, before (I) and after (II) January 1, 1991

\begin{tabular}{lccccc}
\hline & \multicolumn{3}{c}{$\mathrm{D} 1$} & & \multicolumn{2}{c}{$\mathrm{D} 2$} \\
\cline { 2 - 3 } \cline { 5 - 6 } \cline { 5 - 6 } & $\mathrm{I}(n=126)$ & $\mathrm{II}(n=254)$ & & $\mathrm{I}(n=131)$ & $\mathrm{II}(n=200)$ \\
\hline Compliance & 13.5 & 22.4 & & 16.0 & 20.0 \\
Minor non-compliance & 69.8 & 63.0 & & 48.8 & 60.0 \\
Major non-compliance & 16.7 & 14.6 & & 35.2 & 20.0 \\
Overall non-compliance & 86.5 & 77.6 & & 84.0 & 80.0 \\
No contamination & 68.3 & 77.2 & & 63.4 & 76.5 \\
Minor contamination & 31.7 & 21.7 & & 36.6 & 23.5 \\
Major contamination & 0.0 & 1.2 & & 0.0 & 0.0 \\
Overall contamination & 31.7 & 22.9 & & 36.6 & 23.5 \\
\hline
\end{tabular}

All values are percentages

D2 patients. In $65.3 \%$ of the D1 patients with noncompliance this was restricted to 1 or 2 stations (minor non-compliance) versus $55.6 \%$ in D2. In $15.3 \%$ of the D1 and $25.9 \%$ of the D2 patients non-compliance ranged from 3 to 5 stations, and in D2 non-compliance occurred in up to 11 stations (major non-compliance). Overall, contamination occurred in $25.8 \%$ of the D1 and $28.7 \%$ of the D2 patients (Table 4 ). The magnitude of contamination was modest and equally distributed over D1 and D2. Major contamination occurred in only three D1 patients.

To evaluate the effect of the additional measures taken on January 1 1991, the patients were divided into two groups, those entered before January 1, 1991 (I) and those entered after this date (II). In D1 patients, non-compliance decreased from $86.5 \%$ in the first period to $77.6 \%$ in the second period (Table 5). The magnitude of non-compliance decreased only slightly; minor non-compliance was reduced from $69.8 \%$ to $63 \%$ and major non-compliance was reduced from $16.7 \%$ to $14.6 \%$. In D2 patients, non-compliance decreased from $84 \%$ in the first period to $80 \%$ in the second period, mainly because of reduced major non-compliance.

In D1 patients, contamination decreased from $31.7 \%$ to $22.9 \%$, and in D2 patients from $36.6 \%$ to $23.5 \%$ after the implementation of the additional measures, but the magnitude of contamination, which was already modest, remained unchanged.

The positive effect of the intensification of quality control on lymph node yields was limited: both the total nodal yield per $\mathrm{N}$ level and the variability per $\mathrm{N}$ level improved only slightly (Table 6).

For D2 dissections, the incidence of protocol deviations per referent surgeon is shown in Table 7. Insignificant individual differences were found among referent surgeons for non-compliance $(P=0.10)$, but for contamination the differences among the surgeons were significant $(P<0.001)$. The lowest non-compliance score $(42.9 \%)$ was achieved by the Japanese referent surgeon, when he retrieved the lymph nodes himself. With respect to contamination, he ranked first among fellow referent surgeons (55.6\%), but, similarly to the non-compliance results, when he did the lymph 
node retrieval himself, contamination decreased, to $28.6 \%$.

The distribution of lymph nodes in the stations defined by the JRSGC is shown in Table 8 . Comparison of the yields obtained in our trial with those obtained in an experimental study of total gastrectomy and extended lymph node dissection in cadavers showed the most important difference to be in station 4 (greater curvature) [11]. Considering that this is the largest station, the mean value (1.2) in the experimental study is quite low. Even in patients with optimal lymph node dissection, all stations sometimes contain no lymph nodes at all.

The influence of protocol deviations on hospital morbidity and mortality is listed in Table 9. Neither non-compliance nor contamination was associated with increased complication or mortality rates. The 5-year survival curves for patients with and without protocol

Table 6. Distribution of lymph node yields before (I) and after (II) January 1 1991; curative patients $(n=708$; missing, 3)

\begin{tabular}{|c|c|c|c|c|c|c|}
\hline & \multicolumn{6}{|c|}{ N1 } \\
\hline & \multicolumn{3}{|c|}{ I } & \multicolumn{3}{|c|}{ II } \\
\hline & $n$ & Mean & $\mathrm{SD}$ & $n$ & Mean & $\mathrm{SD}$ \\
\hline D1 & 125 & 13 & 8 & 254 & 17 & 10 \\
\hline \multirow[t]{4}{*}{ D2 } & 130 & 16 & 10 & 199 & 16 & 9 \\
\hline & \multicolumn{6}{|c|}{$\mathrm{N} 2$} \\
\hline & \multicolumn{3}{|c|}{ I } & \multicolumn{3}{|c|}{ II } \\
\hline & $n$ & Mean & SD & $n$ & Mean & SD \\
\hline D1 & 125 & 2 & 3 & 254 & 1 & 2 \\
\hline D2 & 130 & 12 & 9 & 199 & 13 & 7 \\
\hline
\end{tabular}

deviations, depicted in Fig. 2 (for non-compliance) and Fig. 3 (for contamination) show that protocol deviations did not influence survival, although there was a nonsignificant survival advantage for patients with complete compliance.

\section{Discussion}

The standardization of surgery-pathology team performance in lymph node dissection is a major problem. The clinical status of the patient, the extent of tumor growth, the operating strategy preferred by the surgeon, and the scrutiny of the pathologist in assessing the resection specimens all affect the team performance. Theoretically, D1 and D2 dissection can be defined strictly, but

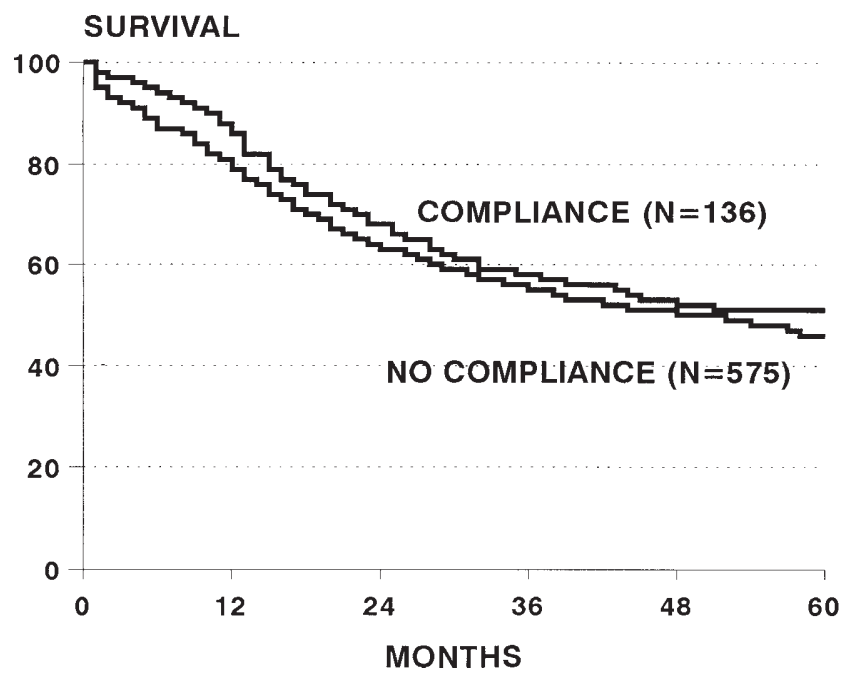

Fig. 2. Survival curves for compliance and non-compliance in curative patients $(n=711)$

Table 7. Incidence of protocol deviations per referent surgeon; curative D2 patients $(n=331)$

\begin{tabular}{lccc}
\hline Referent surgeon & $\begin{array}{c}\text { Number of } \\
\text { patients }\end{array}$ & $\begin{array}{c}\text { Minor and major } \\
\text { non-compliance (\%) }\end{array}$ & $\begin{array}{c}\text { Minor and major } \\
\text { contamination (\%) }\end{array}$ \\
\hline 1 & 30 & 70.0 & 23.3 \\
2 & 23 & 87.0 & 30.4 \\
3 & 61 & 83.6 & 37.7 \\
4 & 38 & 86.8 & 44.7 \\
5 & 31 & 93.5 & 25.8 \\
6 & 29 & 79.3 & 10.3 \\
7 & 36 & 83.3 & 11.1 \\
8 & 49 & 79.6 & 18.4 \\
M.S. & 27 & 77.8 & 55.6 \\
M.S. (+ retrieval) & 7 & 42.9 & 28.6 \\
& & $P=0.10$ & $P<0.001$ \\
\hline
\end{tabular}

a The Japanese instruction surgeon M.S. retrieved the lymph nodes himself in seven patients 
Table 8. Lymph node yields per station. (as defined by the Japanese Research Society for the study of Gastric Cancer (JRSGC) [7]

\begin{tabular}{|c|c|c|c|c|c|c|}
\hline \multirow[b]{2}{*}{ Lymph node station (no.) } & \multicolumn{3}{|c|}{$\operatorname{DGCT}(n=711)$} & \multicolumn{3}{|c|}{$\begin{array}{l}\text { Wagner values } \\
(n=30)[11]^{\mathrm{a}}\end{array}$} \\
\hline & Mean & Range & SD & Mean & Range & SD \\
\hline Right para-cardia (1) & 3.4 & 0-18 & 3 & 2.2 & $0-4$ & 0.9 \\
\hline Left para-cardial (2) & 2.2 & $0-10$ & 3 & 2.0 & $0-5$ & 1.1 \\
\hline Lesser curvature (3) & 5.3 & $0-36$ & 4 & 4.5 & $0-14$ & 3.1 \\
\hline Greater curvature (4) & 5.3 & $0-42$ & 4 & 1.2 & $0-7$ & 1.6 \\
\hline Suprapyloric (5) & 0.8 & $0-17$ & 1 & 2.2 & $0-5$ & 1.1 \\
\hline Infrapyloric (6) & 4.0 & $0-17$ & 3 & 2.7 & $0-8$ & 1.8 \\
\hline Left gastric artery (7) & 3.0 & $0-17$ & 2 & $2.3^{*}$ & $0-5$ & 2.0 \\
\hline Hepatic artery $(8)$ & 2.7 & $0-15$ & 2 & $2.3^{*}$ & $0-6$ & 2.0 \\
\hline Celiac axis (9) & 3.0 & $0-13$ & 2 & 4.2 & $0-15$ & 3.3 \\
\hline Splenic hilus (10) & 1.7 & $0-12$ & 2 & 2.2 & $0-6$ & 1.3 \\
\hline Splenic artery (11) & 2.3 & $0-21$ & 3 & 2.0 & $0-9$ & 2.0 \\
\hline Total (1-11): & & & & & & \\
\hline Distal gastrectomies & 20.4 & $0-86$ & 13 & - & - & 一 \\
\hline Total gastrectomies & 26.4 & $0-105$ & 15 & 27 & $17-44$ & 6.0 \\
\hline
\end{tabular}

* Stations 7 and 8 were combined (mean, 4.6; range, 1-11); the values here are estimations

${ }^{a}$ See text for brief description of Wagner's study

Table 9. Protocol deviations and complications in curative patients $(n=711)$

\begin{tabular}{lcccc}
\hline & $\begin{array}{c}\text { Non- } \\
\text { compliance }\end{array}$ & Compliance & Contamination & $\begin{array}{c}\text { No } \\
\text { contamination }\end{array}$ \\
\hline Total number & 576 & 135 & 193 & 518 \\
Complications & $192(33.3 \%)$ & $44(32.6 \%)$ & $66(34.2 \%)$ & $170(32.8 \%)$ \\
Hospital mortality $^{\mathrm{a}}$ & $43(7.5 \%)$ & $4(3.0 \%)$ & $12(6.2 \%)$ & $35(6.8 \%)$ \\
\hline
\end{tabular}

Differences in complications and hospital mortality between patients with and without protocol deviations are not significant

${ }^{\text {a }}$ Hospital mortality is defined as death within 30 days of operation or during hospital stay

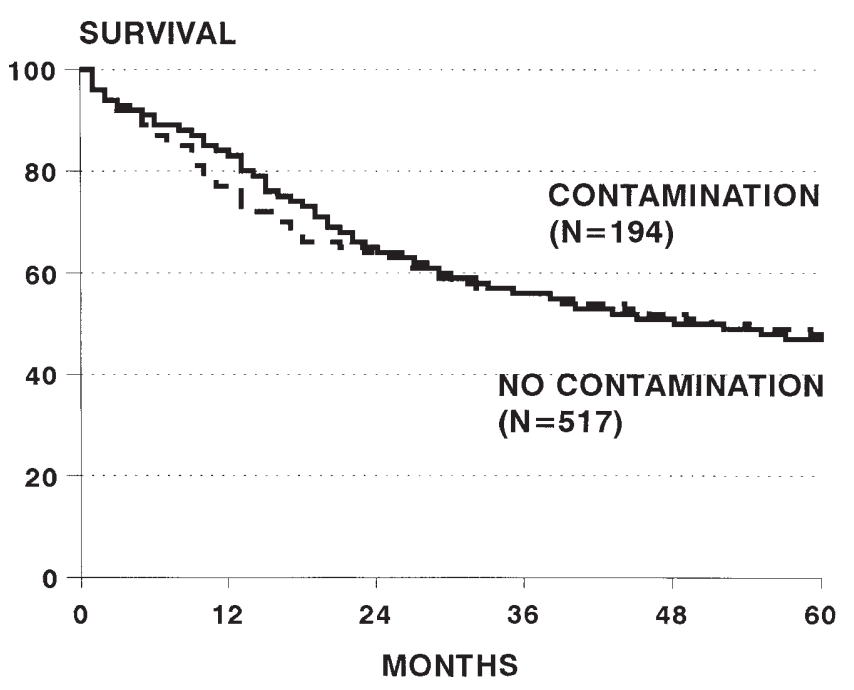

Fig. 3. Survival curves for contamination and no contamination in curative patients $(n=711)$ in practice there is some variation in the extent of nodal clearance depending on the technical ability to dissect the indicated lymph node stations.

Randomized controlled trials comparing two surgical techniques are still relatively rare, and thus far, parameters to measure the level of standardization are not available. In a previous report, protocol adherence was evaluated by estimation of non-compliance (i.e., no proof of lymphadenectomy of indicated stations) and contamination (i.e., proof of a more extensive lymph node dissection than intended) [12]. The incidence of non-compliance in D2 dissection (84\%) and of contamination in D1 dissection (48\%) in this previous report was prominent and suggested a tendency to perform intermediate-type dissections.

For correct interpretation of the quality control parameters, identification of possible sources of error in these parameters is necessary; in Table 10 these sources are listed. Incorrect assessment of the location of the 
Table 10. Possible sources of errors in parameters used to evaluate quality of lymph node dissection

\begin{tabular}{|c|c|c|c|}
\hline Event & Action & Sources of error & Influence $^{a}$ \\
\hline \multirow[t]{7}{*}{ Laparotomy } & Assessment of site & Preoperative evaluation & Both \\
\hline & & Assessment by palpation & Both \\
\hline & & Interpretation of JRSGC criteria & Both \\
\hline & Dissection & Biopsy of suspected nodes & Contamination \\
\hline & & Infiltrating tumor & Contamination \\
\hline & & Splenectomy for bleeding & Contamination \\
\hline & Dissection & Incomplete clearance & Non-compliance \\
\hline \multirow[t]{7}{*}{ Preparation of specimen } & Division of stations & Anatomical variations & Both \\
\hline & & Amount of fatty tissue & Both \\
\hline & & Identification of borders & Both \\
\hline & Lymph node retrieval & Amount of fatty tissue & Non-compliance \\
\hline & & Size of lymph nodes & Non-compliance \\
\hline & & Diligence & Non-compliance \\
\hline & & Clearance techniques & Non-compliance \\
\hline Histological examination & Examination & Incomplete examination & Non-compliance \\
\hline Report & Trial forms & Administrative & Non-compliance \\
\hline \multirow[t]{2}{*}{ Analysis } & Statistical calculation & Assumptions & Both \\
\hline & & Calculations & Both \\
\hline
\end{tabular}

${ }^{\mathrm{a}}$ Errors may cause non-compliance, contamination, or both

primary tumor and misinterpretation of the criteria of the JRSGC may induce general deviations, but contamination is almost always related to surgical management. It is partly caused by extension of surgery due to technical reasons (infiltrative tumor growth, splenectomy for bleeding from the spleen), but also reflects the tendency to augment local clearance when there are suspected (lymph node) metastases. The magnitude of contamination by the Japanese referent surgeon is explained by the definition of lymph node stations, which was slightly altered on his advice after the instruction period.

It should be realized that contamination in D1 patients does not necessarily mean that D2 dissections were performed. The intensive quality control by regional referent surgeons and the coordinator prevented mixing of dissection types and none of the D1 patients underwent en-bloc lymphadenectomy of the N2 level (i.e. front leaf of the mesocolon, pancreas capsula, and clearance of the right part of the hepatoduodenal ligament).

Non-compliance, on the other hand, occurs most prominently during processing of the specimen. This is confirmed by the results of protocol deviations in D2 patients (Table 7), in whom the variation among the surgeons was non-significant, but retrieval of lymph nodes by the Japanese referent surgeon himself markedly reduced non-compliance. It appears that noncompliance is not a reflection of the tendency to perform intermediate resections, but rather, an indication of the quality of processing.
The assumptions made in the model induce errors as well. The reference values of lymph node retrieval were obtained from resections in cadavers, and lymph node clearance techniques were used to obtain maximum yields [11]. Japanese data for numbers of lymph nodes per station indicate that there is a biological variability, and sometimes small stations (numbers 2, 5, and 10) may not contain lymph nodes at all [1]. This is confirmed by our data, which show minimal values of yields upon pathological examination in stations 2, 5, and 10 (Table 8). This illustrates that biological variation is an important cause of non-compliance. The substantial difference in lymph node yields in station 4 is likely caused by different anatomical definitions of this station, because it is the largest station, surrounding the entire greater curvature.

Compared to the initial evaluation, the incidence of protocol deviations was reduced, most markedly in contamination [12]. This reduced incidence was caused mainly by adjustment of the model according to practical applications of the criteria of the JRSGC. Within the protocol of the trial, total gastrectomy was allowed for tumors in the middle part of the stomach with extension towards the cardia. Furthermore, the possibility of performing proximal gastrectomy was excluded from the model, because this procedure has been abandoned in The Netherlands. These adjustments had their greatest impact on contamination, since two restrictions for total gastrectomy in the initial model were lifted.

The reduction of the systematic errors in the adjusted model provides better insight into the real 
protocol deviations, and in this respect, the validity of the model increased. On the other hand, the original model demonstrated more clearly the limitations of the rather theoretical nature of the JRSGC criteria.

The monitoring of protocol adherence during the intake phase of pragmatic clinical trials and the implementing of measures to increase standardization of the surgery-pathology team performance may preserve the validity of trials [8]. Therefore, during the remaining intake period of our trial, measures were taken to minimize non-compliance and contamination. Upon implementation of the measures, the incidence of noncompliance and contamination decreased in D1 as well as in $\mathrm{D} 2$ dissections (Table 5), although the reduction in contamination was most marked. Major protocol deviations persisting in the second period were seen only in non-compliance and varied around the $15 \%$ level for both D1 and D2 operations. The remaining minor deviations should probably be regarded as within acceptable margins of variability in lymphadenectomy, and reflect the complexity of attaining high levels of standardization in multicenter trials. Our conclusion that implementation of the measures improved standardization of the surgery-pathology team performance may be tempered by the presence of a spontaneous 'learning effect'. As the trial progressed, experience with the techniques gained by the participating surgerypathology teams increased, and this may have contributed to the observed improvements in protocol adherence, irrespective of the measures taken. However, the lack of an increase in yields after the intensifying of quality control illustrates that this 'learning effect' probably did not occur (Table 6).

Variations in the 'radicality' of surgery are reported to be associated with hospital complications and longterm survival [13]. These variations are often used as an explanation for the lack of benefit of adjuvant therapy in randomized trials. Indeed, one of the rare trials with a significantly increased survival for the chemotherapy group required precise assessment of surgical procedures and accepted only patients with accurate documentation of curative resection [14]. Although protocol deviations were detected in a substantial proportion of the patients in our trial, there was no association with increased risk of complications or reduced survival. The slight survival advantage in patients with complete compliance does not indicate that complete node dissection improves survival, because, as demonstrated, noncompliance is related to processing of the specimen rather than to surgical management.
Non-compliance and contamination, or the ability to comply with standard criteria of lymphadenectomy, are most reliably determined during surgery. The occurrence of these deviations upon pathological examination requires careful interpretation and optimal knowledge of the processes surrounding the operation. Imperfect as they may be, these deviations represent the only measurable parameters for quality control of surgery outside the operating room at present.

\section{References}

1. Maruyama K, Okabayashi K, Kinoshita T. Progress in gastric cancer surgery and its limits of radicality. World J Surg 1987;11:418-26.

2. Cuschieri A, Fayers P, Fielding J, Craven J, Bancewicz J, Joypaul V, Cook P. Postoperative morbidity and mortality after D1 and D2 resections for gastric cancer: Preliminary results of the MRC randomized controlled surgical trial. Lancet 1996;347:995-9.

3. Bonenkamp JJ, Van de Velde CJH, Songun I, Welvaart K, Sasako M, Hermans J, for the Dutch Gastric Cancer Group. Randomized comparison of morbidity after D1 and D2 dissection for gastric cancer in 996 Dutch patients. Lancet 1995;345:745-8.

4. Pacelli F, Doglietto GB, Bellantone R, Alfieri S, Sgadari A, Crucitti F. Extensive versus limited lymph node dissection for gastric cancer: A comparative study of 320 patients. Br J Surg 1993;80:1153-6.

5. Roder JD, Böttcher K, Siewert JR, Busch R, Hermanek P, Meyer H-J and the German Gastric Carcinoma Study Group. Prognostic factors in gastric carcinoma. Results of the German Gastric Carcinoma Study 1992. Cancer 1993;72:2089-97.

6. Smith JW, Shiu MH, Kelsey L, Brennan MF. Morbidity of radical lymphadenectomy in the curative resection of gastric carcinoma. Arch Surg 1991;126:1469-73.

7. Kajitani T. Japanese Research Society for the Study of Gastric Cancer. The general rules for gastric cancer study in surgery and pathology. Jpn J Surg 1981;11:127-45.

8. Buyse M. Randomized clinical trials in surgical oncology. Eur J Surg Oncol 1991;17:421-8.

9. Sasako M, Maruyama K, Kinoshita T, Bonenkamp JJ, Van de Velde CJH, Hermans J. Quality control of surgical technique in a multicenter, prospective, randomized, controlled study on the surgical treatment of gastric cancer. Jpn J Clin Oncol 1992;22:41-8.

10. Hermanek P, Sobin LH, editors. UICC: TNM classification of malignant tumours, 4th ed. Berlin: Springer Verlag; 1987.

11. Wagner PK, Ramaswamy A, Ruschoff J, Schmitz-Moormann P, Rothmund M. Lymph node counts in the upper abdomen: Anatomical basis for lymphadenectomy in gastric cancer. Br J Surg 1991;78:825-7.

12. Bunt AMG, Hermans J, Boon MC, van de Velde CJH, Sasako M, Fleuren GJ, Bruijn JA. Evaluation of the extent of lymphadenectomy in a randomized trial of Western versus Japanese type surgery in gastric cancer. J Clin Oncol 1994;12:417-22.

13. McArdle CS, Hole D. Impact of variability among surgeons on postoperative morbidity and mortality and ultimate survival. BMJ 1991;302:1501-5.

14. Controlled trial of adjuvant chemotherapy following curative resection for gastric cancer. The Gastrointestinal Tumor Study Group. Cancer 1982;49:1116-22. 